\title{
Systemic Lupus Erythematosus with Severe Nephritis That Mimicked Henoch-Schoenlein Purpura
}

\author{
Ibrahim Al-Attrach ${ }^{* 1}$, Amar Al-Shibli ${ }^{1}$, Layla Al-Riyami1 ${ }^{1}$, Suhail Al-Salam² \\ 1. Division of Nephrology, Department of Pediatrics, Tawam Hospital, Al-Ain, United Arab Emirates \\ 2. Department of Pathology, Faculty of Medicine and Health Sciences, Al-Ain, United Arab Emirates
}

\begin{abstract}
Introduction: Systemic lupus erythematosus (SLE) belongs to a family of related autoimmune rheumatic disorders that are capable of affecting multiple organs, and they are all associated with a variety of autoantibodies. Henoch Schoenlein purpura (HSP) is a sort of systemic vasculitis that is not associated with auto-antibodies and can affect different organs including the kidneys.
\end{abstract}

Case report: A 12 year-old girl presented with abdominal pain, low grade fever, swollen and tender feet and left hand, skin rash on the lower extremities, and high blood pressure. Initial laboratory tests revealed severe proteinuria, microscopic hematuria and low C3 level. Renal biopsy showed diffuse proliferative glomerulonephritis with IgA, fibrinogen and $\mathrm{C} 3$ deposits. The case was accordingly diagnosed as HSP with severe IgA nephropathy. Treatment was started with mycophenolate mofetil (MMF) and pulse methylprednisolone followed by prednisolone. The patient improved and treatment was discontinued after 5.5 months. One month after withdrawal of her medications, the patient presented again with serositis and recurrent proteinuria. Both antinuclear antibodies (ANA) and anti dsDNA were positive. At this point she was diagnosed to have SLE disease and immunosuppressive treatment was restarted. Following this, symptoms disappeared, proteinuria regressed and anti-dsDNA titer dropped.

Conclusion: This case presented with features of HSP and was later-on diagnosed to have SLE. This kind of clinical overlapping has not been reported in the literature to the best of our knowledge.

\footnotetext{
* Corresponding Author; Ibrahim Al-Attrach, MD, Division Chief and Consultant, Pediatric Nephrology, Tawam Hospital, P.O. Box 15258, Al Ain, United Arab Emirates; E mail: iattrach@gmail.com
}

Keywords: Henoch-Schoenlein Purpura; Nephritis; Systemic Lupus Erythematosus

\section{The authors declared no conflict of interest}

\section{Introduction}

Systemic lupus erythematosus (SLE) belongs to a family of related autoimmune rheumatic disorders, including dermatomyositis, scleroderma, polymyositis, mixed connective tissue disease, and rheumatoid arthritis. All of these disorders are capable of affecting multiple organs, and they are all associated with a variety of autoantibodies that rise and fall depending on the clinical disease pattern and/or the treatment given. Because symptoms in systemic rheumatic diseases often overlap, the diagnosis of SLE is not always clear-cut and true overlapping has been frequently reported in the literature. Henoch Schoenlein purpura (HSP) is a sort of systemic vasculitis that is not associated with auto-antibodies and can affect different organs including the kidneys. Its diagnosis is mainly clinical. We report on a case that presented with "typical" clinical features of Henoch-Schoenlein purpura with renal biopsy features of IgA nephropathy. At a later stage the full blown features of systemic lupus erythematosus became apparent.

\section{Case report}

A 12 year-old previously healthy girl, was referred to our hospital with a history of recent symptoms including abdominal pain, low grade fever, swollen and tender feet and left hand, skin rash on the lower extremities, left-sided neck pain and vomiting. On presentation her general condition was stable but her blood pressure was $138 / 100 \mathrm{mmHg}$ and her temperature $37.8 \mathrm{C}$. Her body weight was $61 \mathrm{~kg}$ and her body surface area (BSA) was $1.6 \mathrm{~m}^{2}$. On admission she had purpuric, purple-colored 
skin rash on the lower extremities, no pitting edema, no hepatosplenomegaly, no lymphadenopathy or joint swelling. Other systems were unremarkable.

Initial laboratory tests revealed severe proteinuria (6-7 $\mathrm{g} /$ day) and microscopic hematuria $(50 \mathrm{RBCs} / \mu \mathrm{l})$. Serum creatinine was $1.0 \mathrm{mg} / \mathrm{dl}$, albumin $24 \mathrm{~g} / \mathrm{l}$, ESR $17 \mathrm{~mm} /$ hour, hemoglobin $10.8 \mathrm{~g} / \mathrm{dl}$, Platelets $170,000 / \mu 1$, WBC $7500 / \mu 1$; normal coagulation profile, cholesterol $328 \mathrm{mg} /$ dl (high), antistreptolysin-O (ASO) titer 400 units, C3 $0.29 \mathrm{~g} / \mathrm{l}$ (low) and C4 $0.16 \mathrm{~g} / \mathrm{l}$ (lower normal range).

Our primary clinical diagnosis was Henoch-Schoenlein purpura (HSP) with significant renal involvement. Renal biopsy was performed on the third admission day. The examined 29 glomeruli showed diffuse increase in the mesangial cells and matrix, accentuation of glomerular size and increased lobulation of some glomeruli. Immunoflorescent study revealed mesangial and membranous granular immunoreactivity to IgA, $\mathrm{C} 3$ and fibrin, with no immunoreactivity to IgG, IgM or $\mathrm{C} 4$. The conclusion of the renal histology was a diffuse proliferative glomerulonephritis with IgA, fibrinogen and $\mathrm{C} 3$ deposits.

According to the clinical presentation, laboratory results and histopathology findings, the case was diagnosed as HSP with severe glomerulonephritis and nephrotic syndrome. This was based on the pathognomonic clinical symptoms and signs of HSP, and the fact that $10 \%$ of HSP cases can have low C3 complement. Moreover, the IgA deposits in the renal biopsy and the absence of IgG were also supportive of this diagnosis.

Treatment was started with pulse methylprednisolone at a dose of $1 \mathrm{~g} / \mathrm{m}^{2} /$ dose, a total of three doses, followed by prednisolone $60 \mathrm{mg} / \mathrm{m}^{2} /$ day ( $\max 80 \mathrm{mg}$ /day) for four weeks then $40 \mathrm{mg} / \mathrm{m}^{2} / 48$ hours for another four weeks then tapering. Mycophenolate mofetil (MMF) $750 \mathrm{mg}$ twice daily was also started. Additional medications included enalapril and candesartan.

Clinically the purpuric rash lasted two days then started to fade out, even before steroids were started. Four weeks after starting treatment all clinical complaints resolved and C3 and ESR normalized. The proteinuria started to decrease slowly but significantly after three months of treatment. Four months later the proteinuria dropped to $0.6 \mathrm{~g}$ /day, the RBCs in urine to $20 / \mu 1$ and serum albumin increased to $30 \mathrm{~g} / \mathrm{l}$. MMF and Prednisolone were given for a total treatment time of 5.5 months, and then they were tapered-off and discontinued.

One month after withdrawal of her medications, the patient came with chest pain, abdominal pain and mild guarding. Echocardiography revealed minimal pericardial effusion while abdominal and chest ultrasound showed moderate left sided pleural effusion. This was diagnosed as serositis. The lab tests revealed a rise in the proteinuria to $8 \mathrm{~g}$ /day. The other tests were as follows: RBCs in urine $>100 / \mu 1$, ESR 36 mm/hour, Albumin 23 g/l, antinuclear antibodies (ANA) 3+ positive, anti dsDNA 225 (cut off 30 ), and hemoglobin $10.5 \mathrm{~g} / \mathrm{dl}$. Serum albumin then dropped to $19 \mathrm{~g} / 1$.

It was clinically and serologically evident at this point that the patient had SLE disease, with 5 out of 11 criteria of the American Rheumatism Association (ARA).

Immunosuppressive treatment was restarted using pulse methylprednisolone followed by oral prednisolone at a dose of $80 \mathrm{mg}$ daily and MMF $750 \mathrm{mg}$ twice daily. Hydroxychloroquine was also added. After starting this treatment the clinical and biochemical status improved, reflected by the disappearance of symptoms and the regression of proteinuria as well as normalization of $\mathrm{C} 3$, C4 and decrease of anti-dsDNA titer. Ten months later the laboratory tests showed fairly well controlled SLE including 24 hour urine protein of $0.2 \mathrm{~g}$ /day, normal C3 and C4 complement, anti dsDNA $42 \mathrm{IU} / \mathrm{ml}$ and ESR 18 $\mathrm{mm} / \mathrm{hr}$. The urine sediment was almost normal.

\section{Discussion}

To the best of our knowledge this is the first reported case of SLE that clinically presented as HSP with severe renal involvement as nephrotic-nephritic syndrome and later-on developed clinical and laboratory manifestations of SLE with markedly positive serology. Systemic lupus erythematosus (SLE) belongs to a family of related autoimmune rheumatic disorders, including dermatomyositis, scleroderma, polymyositis, mixed connective tissue disease, and rheumatoid arthritis. All of these disorders are capable of affecting multiple organs, and the symptoms in systemic rheumatic diseases often overlap as has been repeatedly reported in the literature [1-9].

In our case, the initial clinical diagnosis of HenochSchoenlein Purpura was clinically confirmed by three different skilled pediatricians. The renal histology was a diffuse proliferative glomerulonephritis with fibrinogen and $\mathrm{C} 3$ deposits which can be a feature of either HSP or SLE nephritis. However, the positive immunostaining for IgA in renal biopsy gave more support to the clinical diagnosis of HSP. The low level of complement C3 has been described occasionally in HSP cases in the literature [10] and deposition of IgA in the kidney are typical finding in HSP nephritis [11].

The initial clinical and laboratory response after starting the intensified immunosuppressive treatment with steroids and MMF was first regarded as one of two 
possibilities: first, the glomerulonephritis responded well to this treatment; second, this could have been a spontaneous improvement of HSP nephritis. Because data from randomized clinical trials are sparse, there is no clear path for treatment recommended for the established renal disease in HSP $[12,13]$.

However, as the patient developed frank clinical and laboratory manifestation of SLE after discontinuation of this treatment, we retrospectively proposed that this could have been a good response of SLE nephritis to immunosuppressive therapy $[14,15]$.

\section{Conclusion}

In conclusion, this case presented with clinical features of Henoch-Schoenlein purpura and later-on presented with findings supporting the diagnosis of systemic lupus erythematosus. To the best of our knowledge, this kind of clinical overlapping has not been reported in the literature so far.

\section{References}

1. Choi DH, Kim HK, Park TI, John BM, Kang SH, Lee YS, Kim TH, Lee UJ, Lee TS, Yoon GO. [A case of anti-LKM 1 positive autoimmune hepatitis accompanied by systemic lupus erythematosus]. Korean J Gastroenterol. 2008 Mar;51(3):190-3.

2. Akiyama Y, Suzuki T, Tanaka M, Kobayashi K, Kagiri T, Ishibashi T, Kitagawa H, Imai F, Hara K, Doi Y. [A case of mixed connective tissue disease developed into overlap syndrome of progressive systemic sclerosis, systemic lupus erythematosus, polymyositis and Sjögren’s syndrome]. Arerugi. 1990 Jun;39(6):542-7.

3. Turkcapar N, Olmez U, Ozer D, Duzgun N, Duman M. A case of overlap syndrome with rheumatoid arthritis, systemic lupus erythematosus, systemic sclerosis and secondary Sjögren's syndrome. Rheumatol Int. 2006 Jul;26(9):841-5.

4. Usta Y, Gurakan F, Akcoren Z, Ozen S. An overlap syndrome involving autoimmune hepatitis and systemic lupus erythematosus in childhood. World J Gastroenterol. 2007 May 21;13(19):2764-7.

5. Ahmed AR, Schreiber P, Abramovits W, Ostreicher M, Lowe NJ. Coexistence of lichen planus and systemic lupus erythematosus. J Am Acad Dermatol. 1982 Oct; 7(4):478-83.

6. Khalidi NA, Ajmani H, Varga J. Coexisting systemic lupus erythematosus and sickle cell disease: a diagnostic and therapeutic challenge. J Clin Rheumatol. 2005 Apr;11(2):86-92.

7. Chandrasekhara PK, Jayachandran NV, Thomas J, Narsimulu G. Systemic lupus erythematosus and dermatomyositis with symptomatic bilateral sacroiliitis: an unusual and interesting association. Mod Rheumatol. 2009;19(1):84-6.

8. Lorber M, Gershwin ME, Shoenfeld Y. The coexistence of systemic lupus erythematosus with other autoimmune diseases: the kaleidoscope of autoimmunity. Semin Arthritis Rheum. 1994 Oct;24(2):105-13.

9. Nachbar F, Korting HC, Hoffmann RM, Kollmann M, Meurer M. Unusual coexistence of systemic lupus erythematosus and primary biliary cirrhosis. Dermatology. 1994;188(4):313-7.

10. Krause I, Garty BZ, Davidovits M, Cleper R, Tamary H, Rosenmann E, Eisenstein B. Low serum C3, leukopenia, and thrombocytopenia: unusual features of henoch-schonlein purpura. Eur J Pediatr. 1999 Nov;158(11):906-9.

11. Ferrario F, Rastaldi MP. Henoch-Schonlein nephritis. J Nephrol. 2005 Nov-Dec;18(6):637-41.

12. Bogdanović R. Henoch-Schönlein purpura nephritis in children: risk factors, prevention and treatment. Acta Paediatr. 2009 Dec;98(12):1882-9.

13. Zaffanello M, Fanos V. Treatment-based literature of Henoch-Schönlein purpura nephritis in childhood. Pediatr Nephrol. 2009 Oct;24(10):1901-11.

14. Mak A, Cheak AA, Tan JY, Su HC, Ho RC, Lau CS. Mycophenolate mofetil is as efficacious as, but safer than, cyclophosphamide in the treatment of proliferative lupus nephritis: a meta-analysis and meta-regression. Rheumatology (Oxford). 2009 Aug;48(8):944-52.

15. Radhakrishnan J, Moutzouris DA, Ginzler EM, Solomons N, Siempos II, Appel GB. Mycophenolate mofetil and intravenous cyclophosphamide are similar as induction therapy for class V lupus nephritis. Kidney Int. 2010 Jan;77(2):152-60. 